\title{
1 Identification of circular RNAs in porcine sperm and their relation to sperm motility
}

2 Marta Gòdia $^{1}$, Anna Castelló ${ }^{1,2}$, Martina Rocco ${ }^{1,3}$, Betlem Cabrera ${ }^{1,2}$, Joan E. Rodríguez-Gil ${ }^{3}$,

3 Armand Sánchez ${ }^{1,2}$ and Alex Clop ${ }^{1,4^{*}}$

4

$5{ }^{1}$ Animal Genomics Group, Centre for Research in Agricultural Genomics (CRAG) CSIC-

6 IRTA-UAB-UB, Campus UAB, Cerdanyola del Vallès (Barcelona), Catalonia, Spain

$7 \quad{ }^{2}$ Unit of Animal Science, Department of Animal and Food Science, Autonomous University

8 of Barcelona, Cerdanyola del Vallès (Barcelona), Catalonia, Spain

$9{ }^{3}$ Unit of Animal Reproduction, Department of Animal Medicine and Surgery, Autonomous

10 University of Barcelona, Cerdanyola del Vallès (Barcelona), Catalonia, Spain

$11{ }^{4}$ Consejo Superior de Investigaciones Científicas (CSIC), Barcelona, Catalonia, Spain.

13 *Corresponding author:

14 Dr. Alex Clop, Animal Genomics Group, Centre for Research in Agricultural Genomics

15 (CRAG) CSIC-IRTA-UAB-UB, Campus UAB, Cerdanyola del Vallès (Barcelona),

16 Catalonia, Spain. Email: alex.clop@cragenomica.es. Phone: +34 935636600 ext 3353 


\section{Abstract}

29 Circular RNAs (circRNAs) are emerging as a novel class of noncoding RNAs which

30 potential role as gene regulators is quickly gaining interest. Although circRNAs have been

31 studied in several tissues and cell types across several animal species, the characterization of

32 the circRNAome in ejaculated sperm remains unexplored. In this study, we profiled the

33 sperm circRNA catalogue in 40 boar samples. A complex population of 1,598 circRNAs was

34 shared in at least 30 samples. The predicted circRNAs presented in general low abundances

35 and were highly tissue-specific. Circa $80 \%$ of the circRNAs identified in the boar sperm were

36 reported as novel. We also constructed a circRNA-miRNA interaction network based on

37 experimental and predictive microRNA (miRNA) binding sites. Moreover, we found

38 significant correlation between the abundance of some circRNAs and sperm motility

39 parameters and confirmed two of these correlations by RT-qPCR in 36 samples with extreme

40 sperm quality. Our study provides a thorough characterization of a novel cell type for the

41 circRNA encyclopedia collection and suggests that circRNAs are potential noninvasive

42 biomarkers for male sperm quality and thus, might also hold potential to predict male

43 fertility.

45 Keywords: sperm, circular RNA (circRNA), RNA-seq, miRNA, sperm motility, sperm 46 quality, swine, male infertility. 


\section{Introduction}

54 Food scarcity is a growing concern for our society due to human population growth

55 and climate change. Swine and poultry are the main sources of meat worldwide and large

56 efforts are being taken to increase their production in a sustainable manner. In this sense,

57 reproductive efficiency is one of the key aspects for the sustainability of animal breeding [1].

58 In addition, due to their similarity in genome sequence, anatomy and physiology, swine is

59 quickly becoming an important model for human bio-medical research [2]. In humans,

60 infertility is an increasing problem in contemporary society, affecting one in twenty males

61 [3], and has become a subject of bio-medical research in swine [4-6]. Unlike in humans,

62 where fertility data is based on few records per person, the swine industry has begun to

63 record fertility traits each time a male is used for artificial insemination (AI). In pig intensive

64 production systems, during its productive life, an AI boar typically inseminates around 1,500

65 sows (Balasch, personal communication). Moreover, the porcine ejaculates are routinely

66 evaluated in the AI centers after every extraction. Thus, large datasets of the reproductive

67 ability of these boars is becoming available. Taken all together, swine becomes a good

68 animal model to study semen quality and male fertility.

69 Sperm motility and kinetic parameters provide an objective and reproducible

70 measurement of semen quality that is automatically assessed by the computer-assisted semen

71 analysis (CASA) system. CASA records the percentage of total motile sperm, curvilinear

72 velocity (VCL), straight line velocity (VSL) and velocity of the sperm cells (VAP) and head

73 frequency based on trajectories of motile sperm. This approach has been commonly used to

74 assess semen quality in animal breeding strategies prior to AI in cattle [7], horse [8] and

75 swine [9-11] where significant correlations between sperm motility and fertility have been

76 found. In humans, this technique is also applied to estimate the in vitro fertilizing potential of

77 the ejaculates used in assisted reproductive treatments [12-14]. 
78 Multiple research efforts have demonstrated that sperm quality parameters and

79 fertility outcomes are related to the presence or absence of sperm RNAs. Different studies

80 have provided evidence that the absence or deregulation of certain RNAs is associated to

81 infertility and/or sperm motility as in human [15], mice [16] and cattle [17]. Studies have

82 focused their research on messenger RNAs (mRNAs) and on different classes of non-coding

83 RNAs. In humans, several microRNAs (miRNAs) have been found to be dysregulated in

84 infertile patients [18]. Similarly in bulls, Capra et al. found differential abundances of some

85 miRNAs and transference RNAs (tRNAs) between high and low motility sperm populations 86 [19].

Circular RNAs (circRNAs) are a novel class of non-coding RNAs with a closed loop

88 structure, mainly formed through pre-mRNA back splicing event [20]. CircRNAs are highly

89 stable in vivo in comparison to their mRNA linear counterparts, because of their circular

90 structure, which confers protection against exonucleases, ribozymes, antisense RNAs and

91 small-interfering RNAs [21, 22]. Expanding views on their biogenesis and function suggests

92 that circRNAs can directly regulate the abundance of their cognate mRNA and can also act as

93 miRNA sponges [23], sequestering these miRNAs and thus impeding their post-

94 transcriptional inhibitory roles on target mRNAs [23]. circRNAs have been identified across

95 several species and tissues including the fruitfly [24], human [25, 26], mice [26, 27] and

96 swine [28, 29]. These studies revealed species-, developmental- and tissue- specific

97 expression patterns.

98 During the last few years, there has been a considerable interest in the potential use of 99 circRNAs as biomarkers for health. Several studies have identified circRNAs which

100 abundances were associated to cancer, aneurysms, hypertension, heart failure, diabetes and

101 arthritis, among others [reviewed in 30]. In addition, there are few reports characterizing

102 circRNAs in reproductive organs and cell types including oocytes, embryo, placental tissue, 
103 granulosa cells, immature spermatogenic cells and testis [reviewed in 31]. Up to date, a small

104 number of studies have assessed the circRNA predictive potential for reproduction outcomes.

105 For example, Chang and colleagues identified circRNAs associated to embryo quality and

106 suggested their role as potential predictors of live birth [32] and Qian et al. identified

107 differentially expressed circRNAs in the placenta of pregnant woman affected with 108 preeclampsia [33].

109 Our group has recently carried a thorough porcine sperm transcriptome analysis [34]

110 and the data, in line with previous studies [reviewed in 35], showed that the majority of the

111 transcripts are highly fragmented and with low abundances. Considering their stability and

112 abundance, circRNAs could hold an important potential as reliable biomarkers for sperm

113 quality and fertility traits. Here, we have characterized the circRNA repertoire of the porcine

114 spermatozoa, assessed their potential role as miRNA sponges and investigated the

115 relationship between their abundance levels and sperm motility.

$117 \quad$ Results

118 Characterization of the sperm circular RNA repertoire

1191,598 potential circRNAs were present and shared in at least 30 of the 40 ejaculates

120 (Supplementary Table 1). The majority of the circRNA species were derived from exonic

121 regions (CDS, 3' and 5' UTR) (82.1\%), while only $13.5 \%$ and $4.4 \%$ circRNAs originated

122 from intergenic and intronic segments, respectively (Figure 1A). Among the exonic

123 circRNAs, most were conformed by less than 4 exons (81.0\%), and just a few (14 circRNAs)

124 contained 10 or more exons (Figure 1B). In addition, most exonic circRNAs (76.9\%) were

125 less than 400 bp long (Figure 1C). RNA abundance across the different circRNAs types was

126 low, with a range between 0.19 and 136.4 CPMs and mean and median values of 2.42 and

1270.89 CPMs, respectively (Supplementary Table 1). The top 20 most abundant circRNAs 
128 (Table 1) involved 15 coding genes and one annotated long non-coding RNA. These genes 129 included CEP63, ATP6VOA2, PPA2, PAIP2 and PAXIP1, which have been linked to sperm 130 related traits and male fertility.

131 Only a small fraction of genes produce more than one circRNA. These are considered 132 hotspot circRNAs genes. The circRNAs from hotspot genes are often produced from different 133 back-splicing events of one exon with several others [29]. We detected 12 genes with 5 or 134 more circRNA isoforms (Table 2). Some of these genes, namely TESK2, SPATA19, PTK2 135 and SLC5A10 are related to sperm function and fertility.

Sperm circRNAs might be involved in epigenetic regulation and spermatogenesis

137 We analyzed the potential roles of the boar sperm circRNAs under the assumption 138 that their function is related to their known mRNA counterpart. Gene Ontology (GO) analysis 139 of the circRNAs host genes revealed an enrichment for epigenetic functions including histone 140 modification (q-val: $5.52 \times 10^{-6}$ ), histone H3-K36 methylation (q-val: $8.65 \times 10^{-3}$ ) and 141 chromatin organization (q-val: $2.16 \times 10^{-8}$ ) (Supplementary Table 2). We also identified 142 significant ontologies in spermatogenesis (q-val: $5.81 \times 10^{-4}$ ), cilium assembly (q-val: $4.15 \mathrm{x}$ $14310^{-3}$ ) and developmental process (q-val: $1.33 \times 10^{-2}$ ), among others (Supplementary Table 2).

\section{The boar sperm have a highly specific circRNAome}

145 We compared our circRNA catalogue with equivalent available datasets [36] from 146 other studies in human (including different brain sections tissues and several cell lines) [26, 147 37-40], mice (several brain segments, cell types and embryonic stem cells) [26, 37] and swine 148 (lung, skeletal muscle, fat, heart, liver, spleen, kidney, ovarium, testis and 5 brain sections) $149[28,29]$. Twenty-four $\%$ and $11.3 \%$ of the boar circRNAs had potential human and mouse 150 orthologs, respectively (Table 3). On the other hand, $20.3 \%$ of the porcine sperm circRNAs 151 were also present in other porcine tissues (Table 3). The tissues showing higher overlap with 152 sperm were testes (11.6\%) and cortex (11.3\%). All the other tissues were clearly less 
153 concordant (Table 3). By comparing this in the opposite direction, the proportion of the

154 circRNAs already annotated in any pig tissue that were also present in our pig sperm list was

$1554.9 \%$. This value was 12 times lower $(0.4 \%)$ when evaluating the human circRNA catalog

156 (Table 3).

157 Sperm circRNAs do not follow an age-dependent pattern

158 We assessed whether sperm circRNAs depicted an age-accumulating profile as has 159 been previously observed in rat testes [41] and rat and mouse brain tissues [41, 42]. All the 160 boars from our dataset were mature ( $\geq 8$ months old) with ages ranging between 9 and 54 161 months of age. Sexual maturity in boars is a process that starts at the age of 8 months and 162 finalizes at the age of 2 years [43]. Thus, we divided the samples in those coming from boars 163 approaching sexual maturity with ages below 2 years old and those produced by mature pigs 164 with ages above 2 years old. There was no significant difference in the number of circRNAs 165 identified (P-value: 0.68, Wilcoxon rank sum test) nor in their RNA abundance (P-value: 166 0.948) between the two groups. We repeated the analysis considering only extreme ages: 167 young ( $\mathrm{N}=4$; between 8.6 and 9.2 month old) and mature ( $\mathrm{N}=4 ; 29.9$ to 54.6 month old). 168 Again, there was no difference in the number of circRNAs identified (P-value: 0.89) or in 169 their abundance (P-value: 0.2).

\section{0 circRNA-miRNA interaction network}

171 To characterize the potential role of the circRNAs as miRNA sponges, we built a co172 expression network including the abundances of the 95 miRNAs and the 1,598 circRNAs 173 identified in the short and total RNA-seq datasets. The association analysis resulted in 2,323 174 significant interactions between the 95 miRNAs and 564 circRNAs. On the other hand, the in 175 silico prediction of miRNA targets in the circRNA sequences, based on sequence 176 complementary, yielded 4,987 potential targets involving all the miRNAs and 1,103 177 circRNAs. To reduce the proportion of false-positives, only the 70 interactions (from 31 
178 miRNAs and 56 circRNAs) that were found shared in both methods were used for network

179 visualization (Figure 2). Most circRNAs (46) presented one miRNA target site with the

180 exception of 10 circRNAs that harbored 2 or more potential targets (Figure 2). As the

181 ssc_cic_08954 and ssc_circ_1454 which can potentially regulate, each, 4 distinct miRNAs

182 (Figure 2). On the other hand, miR-26a and miR-28-5p were predicted to be regulated by 9

183 different circRNAs and 5 different circRNAs regulated miR140-3p and miR-423-5p (Figure

184 2).

185 Correlation of circRNAs with sperm motility and circRNA validation

186179 circRNAs (from 26 intergenic regions, 10 intronic regions and 146 genes)

187 showed significant associations between their abundance and different sperm motility

188 parameters (Supplementary Table 3). More in detail, 28 (2 intergenic, 2 intronic and 24

189 protein coding genes), 94 (7 intergenic, 2 intronic and 81 genic), 35 (6 intergenic, 3 intronic

190 and 26 genic) and 57 (11 intergenic, 3 intronic and 43 genic) circRNAs correlated with the

191 percentage of motile cells, VCL, VAP and VSL, respectively (Supplementary Table 3).

192 To confirm the existence of these circRNAs and their phenotypic correlations we

193 undertook a RT-qPCR and Sanger Sequencing approach. First, we randomly selected 2

194 circRNAs with different RNA abundance levels (75.0 and 10.6 CPM) to test whether we

195 could confirm the bioinformatically predicted circRNAs. The chosen circRNAs were:

196 ssc_circ_1141 (from PTGES3) with 75.0 CPM, and ssc_circ_0670 (from BAZ2B) with 10.6

197 CPM, both also detected in human [36] (hsa_circ_0008137 and hsa_circ_0002463,

198 respectively) and in swine testes [28]. The PCR amplification of the 2 circRNAs resulted in a

199 single electrophoretic band of the expected size (Supplementary Figure 1.A) and Sanger

200 Sequencing confirmed the back-splice junction (Supplementary Figure 2.A-B). We,

201 therefore, confirmed the validity of the RT-PCR and Sanger Sequencing approach to validate

202 the presence of tested circRNAs and the existence of these two circRNAs in the boar sperm. 
203 Then, we used the same approach to confirm the existence of 8 circRNAs selected for 204 their significant correlation with at least one motility trait (Supplementary Table 3). These 205 circRNAs were further selected based on the fact that (i) they were present at high 206 abundances, or (ii) they showed significant correlation with at least 1 trait, or (iii) they had 207 been previously identified in pig [28], human [36] or mice [36]. PCR amplification of 6 of 208 the 8 circRNAs resulted in a band of the expected size (Supplementary Figure 1B) and the 209 Sanger Sequencing validated the expected back-splice junction (Figure 3.A-C; 210 Supplementary Figure 2.C-E). ssc_circ_0839 from PAIP2 did not amplify (data not shown) 211 and ssc_circ_0118 from PDE10A (also identified in pig testes and in human as 212 hsa_circ_0078638) displayed 2 amplification bands (Supplementary Figure 1B). These two 213 circRNAs were discarded for further analysis. Thus, we also confirmed the existence of these 2146 circRNAs.

215 The RT-qPCR levels of these 6 circRNAs were measured in 36 animals presenting 216 extreme and opposite values of sperm motility ( $\mathrm{N}=18$ for each phenotypic distribution tail) 217 from a bank of 300 phenotyped boar ejaculates. None of the 36 samples was included in the 218 RNA-seq study. The two sample groups displayed significant phenotypic differences for all 219 the studied traits: percentage of motile cells (P-value: $2.65 \times 10^{-9}$, Wilcoxon rank sum test), 220 VCL (P-value: $2.20 \times 10^{-10}$ ), VSL (P-value: $3.22 \times 10^{-7}$ ) and VAP (P-value: $3.22 \times 10^{-7}$ ). The 221 RT-qPCR assays presented efficiencies between $99.6 \%$ and $105.2 \%$.

222 Two of the 6 circRNAs showed significant differences between the two sperm 223 motility groups (Figure 3.B). These 2 circRNAs were ssc_circ_1458 from LRBA (P-value: 224 0.049) and ssc_circ_1321 from PAPOLA (P-value: 0.035). A third circRNA, ssc_circ_1132, 225 from LIN7A, showed significant differences between the two motility groups (P-value: 0.008) 226 (Figure 3.B) but in the opposite direction than expected according to the RNA-seq data and 227 was consequently considered as not validated due to inconclusive results. The other 3 
circRNAs, ssc_circ_1101 from KHDRBS3, ssc_circ_0437 from ULK4 and ssc_circ_1061

from ZNHIT6 did not present significant differences between the 2 groups (Figure 3.B).

\section{Discussion}

In this study, we have profiled for the first time, the circRNA repertoire of mature

233 spermatozoa in a mammalian species and its association with sperm motility as a parameter

234 of semen quality. These results provide further evidence and expand the relevance of

235 spermatoza RNAs in sperm biology and quality. circRNAs have been reported as promising

236 potential prognostic and diagnostic biomarkers for human health including, among others,

237 cancer, diabetes, cardiovascular diseases or pre-eclampsia [reviewed in 30]. Here, we provide

238 novel data supporting the potential of circRNAs as biomarkers for the male's reproductive

239 function as reflected by association with sperm motility parameters.

240 We have identified nearly 1,600 boar sperm circRNAs that are robustly present in

241 most samples (at least 30). The sperm circRNA repertoire presented similar genomic circular

242 characteristics such as the proportion of genomic overlap with protein coding or intergenic

243 regions, among others, the number of exons and their length (Figure 1), when compared to

244 data previously reported in other tissues from swine [28], human [44] or rat [41].

245 Nonetheless, the list of sperm circRNAs showed a modest overlap with other tissues and

246 species (Table 3). Not surprisingly, the largest overlap was with porcine testes (11.6\%)

247 probably due to the fact that the spermatogenic lineage including spermatozoa is contained in

248 the male gonads. The proportion of pig sperm circRNAs shared across swine tissues was in

249 general low when compared to the rest of the Sus scrofa libraries (Supplementary Table 4).

250 Although this comparative analysis may be partly influenced by technical differences

251 involving the processing of the tissues and the data between the studies, our results suggest

252 that sperm present high tissue-specificity, with circa $80 \%$ of the circRNAs being present only 
253 in sperm, considerably higher than testes [28], with approximately 65\% testes-specific. On

254 the opposite direction, there was a larger coincidence between the catalogue of around 6,660

255 porcine circRNAs $[28,29]$ and the swine sperm circRNAome $(4.9 \%)$, than between the later

256 and the human archive with over 90,000 circRNAs [36] (0.4\%) (Table 3). This 12x fold

257 difference indicates that circRNAs are not only tissue specific but have also a strong species-

258 specific component.

259 We investigated the functional relevance of sperm circRNAs, under the hypothesis

260 that their function is associated to the host gene. Six (CEP63, ATP6V0A2, PPA2, PAIP2 and

$261 P A X I P 1)$ of the 15 coding genes providing the top 20 most abundant circRNAs (Table 1)

262 have been directly implicated, to sperm related traits and male fertility. CEP63 is engaged in

263 microtubule organization, and mice knockout studies revealed its essential role in male

264 fertility [45]. ATP6VOA2 proteins and transcripts are down-regulated in infertile men [46].

265 PPA2 is located in the mitochondrial membrane and might be involved in the production of

266 ATP, control of molecular processes linked to the launching of sperm capacitation and sperm

267 motility [47-49]. PAIP2 is linked to male sperm maturation and fertility [50] and PAXIP1 is

268 associated with developmental arrest of spermatocytes, testicular atrophy, and infertility in

269 knockout mice [51]. We identified 12 circRNA from hotspot genes producing five or more

270 circRNAs each (Table 2). Some of the hotspot genes were related to sperm function and

271 fertility. TESK2 may play a role in early stages of spermatogenesis [52]; PTK2, which is

272 essential for a embryo development [53]; SPATA19, a gene that is critical for sperm

273 mitochondrial function in relation to sperm motility and fertilization ability [54], and

274 SLC5A10, which protein products may act as water channels in spermatozoa [55].

275 The ontology enrichment analysis of the genes harboring circRNAs pointed towards

276 epigenetic related functions, which are essential in all cell types including chromatin

277 condensation in sperm and the reprogramming of gene expression upon egg fertilization and 
278 during embryo development (Supplementary Table 2). Gene enrichment analysis also

279 signaled towards spermatogenesis and developmental processes, the later also implicating the 280 embryo development related genes, DHX36, IPMK, RICTOR, CDC73 and ANGPT1 which

281 were hosting some of the circRNAs found in our analysis (Supplementary Table 1). These

282 functions are in line with the gene ontologies highlighted in studies analyzing sperm mRNAs

$283[34,35,56]$, thereby providing further basis for the hypothesis that circRNAs may exert their

284 function (regulation of sperm quality and motility in our study) by controlling their cognate 285 linear mRNA.

286 A previous study in rat testes identified a circRNA age-dependent dynamic pattern of 287 expression and suggested a relation between their abundance and function with the male's 288 sexual maturity and spermatogenesis [41]. For this reason, we sought to investigate whether, 289 like in testes, sperm circRNAs - in boar at least - also accumulate through age. Our data 290 highlighted that there is no association between the mature sperm circRNAs and the boar's 291 age. Cells with high proliferation rates, seem to accumulate less circRNAs, possibly due to 292 passive thinning out during proliferation [57]. Spermatogenesis is a process that occurs 293 throughout the male's lifetime in which spermatogonial stem cells (SSCs) undergo 294 continuous cell division and finally differentiate to ultimately become spermatozoon [58]. 295 Thus, the fact SSCs undergo continuous self-renewal and keep proliferating through the 296 male's lifetime may impede the accumulation of circRNAs in these cells and thus explain 297 why no age-dependent pattern of circRNA abundance was found in the sperm of pigs with 298 different ages. It is plausible that due to the continuous production and differentiation of the 299 male germ cell, the sperm circRNAome does not mimic the reproductive performance of the 300 boar, at least once the number of germ cells have stabilized which occurs at the age of 7 301 months old in pigs [59] and until the boar is senile. These results infer that stability of mature 
302 sperm circRNAs might be constant for a period in normozoospermic males and provides

303 further evidence for circRNAs as a noninvasive diagnosis tool for male reproductive diseases.

304 To elucidate the functional relevance of circRNAs as miRNA sponges we built an

305 interaction network (Figure 2). We combined benchwork data of circRNA and miRNA

306 abundances (small and total RNA-seq) and in silico searches of miRNA target sequences in

307 circRNAs with the aim to increase the reliability of the circRNA-miRNA relationship

308 predictions. This network with 70 interactions, contained some interesting circRNAs and

309 miRNAs in relation to semen quality and male fertility. Remarkably, two circRNAs,

310 ssc_circ_0954 and ssc_circ_1454, presented, each, 4 different miRNA target sites. The first,

311 ssc_circ_0954 arises from DCDC2C, a gene identified in the human's sperm flagellum end-

312 piece with a suggested role on microtubule dynamics by acting as a

313 depolymerization/polymerization balancing system [60]. This circRNA regulated among

314 others, miR-361-3p which has been found dysregulated in subfertile men [61], miR-423-5p,

315 altered in oligozoospermic men [62] and miR-28-5p, dysregulated in normozoospermic

316 infertile individuals [18]. The other cirRNA, ssc_circ_1454, is transcribed from MTHFD2L, a

317 mitochondrial isozyme from the folate cycle metabolic pathway [63], a vitamin that has been

318 also related to semen quality and fertility in men [64]. One of the miRNAs targeted by

319 ssc_circ_1454 was miR-16, which was found dysregulated in subfertile men [61]. The

320 network also showed 9 circRNAs that may be regulating miR-28, a miRNA (miR-28-5p) that

321 is dysregulated in normozoospermic infertile individuals [18]. The potential miR-28

322 regulators include ssc_circ_1370, arisen from FAM92A, whose protein may play a role in

323 ciliogenesis [65], implicated in the formation of the sperm flagella and ssc_circ_0002 from

324 WDR7, which is associated to cattle sperm quality [66]. Likewise, 9 circRNAs were predicted

325 to regulate miR-26a, which has been in turn, linked to VCL, VSL and VAP motility

326 parameters in swine [67]. These miR-26a regulatory edges implicate ssc_circ_0361 from 
327 ACTL6A, crucial for embryo development [68] and ssc_circ_1352 from CAGE1, an

328 acrosomal protein with proposed roles in fertility [69]. Other interesting interactions included

329 ssc_circ_0345 from the hotspot gene SLC5A10 (sodium-dependent mannose and fructose

330 transporter) (Table 2), which regulated $\mathrm{miR}-423-5 \mathrm{p}$, a miRNA that was found to be

331 upregulated in oligozoospermic semen [62] and let-7c, which is altered in severe

332 aasthenozoozpermia patients [70]. Altogether, the network involves key genes and miRNAs

333 for male fertility thereby suggesting that circRNAs play a functional role in the male

334 reproductive ability.

335 The potential role of circRNAs in male reproductive traits is further substantiated by

336 the associations between sperm motility and the abundance of some circRNAs in our study.

337 At least 20 of the circRNA host genes implicated in the phenotypic correlations have been

338 previously linked to sperm biology or male fertility (Supplementary Table 3). For example,

339 ssc_circ_0823, which correlated with VCL (p-val = 0.009), is a circRNAs hosted by CAMK4,

340 a gene that has been implicated in sperm motility in humans [71]. ssc_circ_0780, correlated

341 with the percentage of motile cells $(\mathrm{p}$-val $=0.041)$ from $L R G U K$, a gene required for sperm

342 assembly including the growth of the axonome, a structure that is necessary for the flagellar

343 beating in sperm [72]. We successfully tested by RT-qPCR 6 of the 179 circRNAs that

344 showed RNA-seq based significant correlations between their abundance and sperm motility.

345 The results allowed us to validate the correlation with motility for 2 of these 6 circRNAs,

346 ssc_circ_1458 from LRBA and ssc_circ_1321 from PAPOLA. Both were significantly down-

347 regulated in the ejaculates with low sperm motility values (Figure 3.B). LRBA is a gene

348 involved in coupling signal transduction and vesicle trafficking but no link with sperm

349 function or fertility has been made thus far. This gene has been associated to immune-related

350 disorders in humans. ssc_circ_1321 from PAPOLA, has a human ortholog (human circRNA:

351 hsa_circ_0033126) and the host gene is implicated in RNA and ATP binding. Thus, none of 
352 the two host genes have been previously related to sperm function or fertility. Moreover,

353 these 2 circRNAs are not included in the circRNA:miRNA interaction network. However, the

354 association is clear and confirmed by RT-qPCR and we cannot exclude unidentified relevant

355 functions on sperm motility for these two genes. Two other circRNAs were also of high

356 interest and tested as they were within the top 20 most abundant (Table 1) and correlated with

357 sperm motility (Supplementary Table 3). They were ssc_circ_0839 from PAIP2, with crucial

358 roles in spermatogenesis [50] that did not amplify, and ssc_circ_1101 from KHDRBS3, found

359 highly abundant in mice testes [73], which did not present significant differences in RT-

360 qPCR levels between motility groups (Figure 3.B).

361 Interestingly, some circRNAs popped up as relevant in more than one of the analysis

362 carried through the study. For example, ssc_circ_1532 from the hotspot gene SPATA19

363 (Table 2), related to sperm motility and fertility [54], was suggested to regulate miR-99a

364 according to the network analysis (Figure 2). miR-99b has been found deregulated in low

365 motile sperm fractions in bull [19] and in subfertile men [61]. Another circRNA,

366 ssc_circ_1219 from OSBPL9, involved in male reproduction [74], displayed abundance

367 correlation with VCL (P-value: 0.04) (Supplementary Table 3) and was identified as a

368 potential target of miR-101 (Figure 2), a miRNA (miR-101-3p) that was altered in 369 asthenozoospermia men [70].

370 Remarkably, 4 (DENND1B, PTK2, SLC5A10 and CAMSAP1) of the 12 hotspot genes

371 hosted a circRNA with significant abundance correlation with sperm motility. Thus, one third

372 of the hotspot genes included circRNAs correlated with motility whilst only $14.9 \%$ of the

373 genes (147) of the 984 genes hosting the 1,598 circRNAs were correlated to motility.

374 Noteworthy, the 12 circRNA hotspot genes were not hotspots in the other porcine tissues

375 analyzed [28, 29] (data not shown). Altogether, this indicates that circRNAs hotspot genes

376 may have relevant tissue-specific functions. 

different levels, that there is a detectable population of circRNAs in the boar sperm that is

379 related to relevant functions in sperm and fertility. Moreover, these circRNAs are stable

380 across age. For these reasons, our results indicate that circRNAs hold a potential as

381 biomarkers for sperm motility and potentially, fertility outcomes [7, 8, 10-14, 75]. This

382 potential should be further explored in swine, as well as in other domestic animals and in 383 human fertility clinics.

384 In conclusion, our study is the first to characterize the spermatozoa circRNAs 385 repertoire in an animal species. We have provided a comprehensive view of the boar sperm 386 circRNAome and their correlation with sperm motility parameters. Our findings involving 387 sperm motility may spur novel research on male fertility in both human medicine and in 388 animal breeding.

\section{Material and methods}

Sperm collection, phenotyping and library preparation

Ejaculates from 300 Pietrain boars were collected using the hand glove method by

393 trained professionals at commercial farms. Fresh sperm motility traits were assessed with the

394 CASA system (Integrated Sperm Analysis System V1.0; Proiser, Valencia, Spain). In this 395 study we analyzed sperm motility parameters, including the total percentage of motile cells, $396 \operatorname{VCL}(\mu \mathrm{m} / \mathrm{s})$, VSL $(\mu \mathrm{m} / \mathrm{s})$ and VAP $(\mu \mathrm{m} / \mathrm{s})$. The average percentage of motile cells was 75.1 397 with a standard deviation (SD) of 18.3, VCL (mean: 45.1; SD: 12.6), VSL (mean: 27.0; SD: 398 8.3) and VAP (mean: 34.2; SD: 10.5). Phenotypes were corrected for the fixed variables: 399 farm (1, 2, 3), age (1, 2, 3) and season and year (Autumn 2014, 2015 and 2016; Winter 2015, 4002016 and 2017; Spring 2015 and 2016; Summer 2015) using the R function "Im" [76]. 401 Ejaculates were purified to remove somatic cells and immature sperm cells and purified 
402 sperm was stored at $-96^{\circ} \mathrm{C}$ with Trizol ${ }^{\circledR}$ as described by Gòdia et al. [77]. RNA was extracted 403 from purified sperm cells, treated with TURBO DNA-free ${ }^{\mathrm{TM}}$ Kit (Invitrogen; Carlsbad, USA)

404 and quantified using Qubit ${ }^{\mathrm{TM}}$ RNA HS Assay kit (Invitrogen; Carlsbad, USA). The RNA 405 yield of these samples averaged $2.2 \mathrm{fg}$ per sperm cells (the range was between 0.8 and 3.7 $406 \mathrm{fg}$ ). We assessed RNA integrity with the 2100 Bioanalyzer using the Agilent RNA 6000 Pico 407 kit (Agilent Technologies; Santa Clara, USA). All samples presented RNA Integrity Number 408 (RIN) below 2.5, which indicates the absence of intact RNA from somatic cell origin. We 409 then performed RT-qPCR assays for PRMI and PTPRC mRNAs as well as for 410 intergenic/genomic DNA to verify that all the samples were free from RNA from somatic 411 cells and from genomic DNA contamination [77].

41240 sperm RNA samples were subjected to total RNA-seq. 34 of these samples were 413 also used for small RNA-seq. For total RNA-seq libraries, ribosomal RNA (rRNA) was 414 depleted with the Ribo-Zero Gold rRNA Removal Kit (Illumina) and libraries were 415 constructed with the SMARTer Universal Low Input RNA library Prep kit (Clontech). 416 Resulting libraries were sequenced in a HiSeq2500 system (Illumina) to generate 75 bp long 417 paired-end reads. For small non coding RNA-seq libraries, extracted RNA without rRNA 418 depletion was directly subjected to library preparation with the NEBNext Small RNA Library 419 Prep Set kit (New England Biolabs) and sequenced on a HiSeq2000 (Illumina) to generate 50 420 bp single-end reads.

\section{$421 \quad$ RNA-seq and bioinformatic analysis}

422 For the total RNA fraction, we obtained, in average, 20.4 million paired-end reads per 423 sample. Raw reads were then filtered by removing adaptor sequences and low-quality reads 424 with Trimmomatic v.0.36 [78]. In average, $98.5 \%$ of these reads passed the quality control 425 filters. The identification of circRNAs was carried on these reads with the find_circ pipeline 426 [26] with stricter filter stringency. To reduce the false positive rate in the discovery of 
427 circRNAs, we selected circular splice transcripts with at least two unique supporting reads in

428 the anchor segment and with Phred quality scores of 35 or more. Moreover, only circRNAs

429 predicted in at least 30 samples were kept. The RNA abundance of the predicted circRNAs

430 were normalized as the number of back-splice junction spanning reads by its sequencing

431 depth, as counts per million (CPM). The functional regions of circRNAs were identified

432 based on their co-location with genomic features (e.g. exon, 3'UTR, 5'UTR, etc) from the

433 Ensembl database (release 91) with BEDtools [79]. Our catalogue of boar sperm circRNAs

434 was contrasted with other publically available porcine circRNA databases including heart,

435 liver, spleen, lung, kidney, ovary, testis, skeletal muscle, fat and fetal brains [28, 29]. We also

436 queried several human tissues (including several cell lines, brain sections placenta, muscle,

437 fat, umbilical cord, atrium, decidua and plasma) [26, 37-40] and murine (cell lines and brain

438 sections) [26, 37] available at the circBase database [36]. Genomic coordinates from the

439 human and mouse circRNAs were liftover to Sscrofa11.1 using the UCSC liftover tool [80].

440 For the small RNA-seq analysis, we obtained an average of 7.3 million reads per

441 sample. Trimming of adaptors and low quality bases was performed with Cutadapt v1.0 [81].

$44299.2 \%$ of these reads were of high quality and were thus used for downstream analysis. The

443 mapping of sncRNAs was performed with the sRNAtoolbox v.6.17 [82] with default settings

444 and providing miRBase [83] release 21 as library dataset. Multi-adjusted read counts were

445 then normalized by sequencing depth as CPM. We only considered the miRNAs that were

446 detected $>1 \mathrm{CPM}$ in all the samples.

447 circRNAs-miRNAs network visualization

448 For a functional annotation of the circRNA and miRNA interactions in a systems 449 biology context, we carried a Partial Correlation with Information Theory (PCIT) analysis 450 [84] using the combined list of circRNAs and miRNAs after normalization of abundance 
451 levels with $\log 2$. We further assessed circRNAs-miRNAs co-abundance interactions using

452 miRanda [85] v.3.3a. Shared correlations were visualized with Cytoscape v.3.7.0 [86].

453 Genome Ontology analysis and correlation with sperm motility parameters

454 GO analysis was carried with PANTHER v.13.1 [87] with the overrepresentation test 455 and p-values corrected with FDR. Annotation Data Set was "GO biological process 456 complete". Pearson correlation was used to determine associations between circRNA 457 abundance levels and sperm motility parameters. P-values $<0.05$ were considered 458 statistically significant.

Validation of circRNAs and reverse transcription quantitative PCR (RT-qPCR)

divergent primers. Primers were designed as in [88] using the Primer Express software 462 (Applied Biosystems). Primer sequences are shown in Supplementary Table 5. For cDNA 463 synthesis, $5 \mu$ of RNA were reverse transcribed using the High Capacity cDNA Reverse 464 Transcription kit in a final volume of $50 \mu \mathrm{L}$ (Applied Biosystems; Waltham, USA) following 465 the manufacturer's protocol. circRNAs were amplified and visualized in 3\% high resolution 466 agarose gel electrophoresis and confirmed by Sanger Sequencing.

467 The abundance level of 6 circRNAs, correlated to sperm motility parameters in the 468 RNA-seq study, was analyzed by RT-qPCR in 36 samples, none of them included in the 469 RNA-seq. These 36 samples belong to two groups with extreme and divergent values for 470 sperm motility from a bank of 300 ejaculates with phenotypic records. Quantitative PCR 471 reactions were performed in triplicate in $15 \mu \mathrm{L}$ final volume including $7.5 \mu \mathrm{L}$ SYBR Select 472 Master Mix (Life Technologies - Thermo Fisher Scientific), $300 \mathrm{nM}$ of each primer and 3.75 $473 \mu \mathrm{L}$ of cDNA 1:4 diluted on a QuantStudio 12K Flex Real-Time PCR System (Applied 474 Biosystems). To evaluate the efficiency of the RT-qPCR assays, standard curves with 6 serial 475 dilutions from a pool of sperm cDNA were generated. Thermal profile was set as follows: 
$47650^{\circ} \mathrm{C}$ for $2 \mathrm{~min}, 95^{\circ} \mathrm{C}$ for $10 \mathrm{~min}$ and 40 cycles at $95^{\circ} \mathrm{C}$ for $15 \mathrm{sec}$ and $60^{\circ} \mathrm{C}$ for $60 \mathrm{sec}$.

477 Moreover, a melting profile $\left(95^{\circ} \mathrm{C}\right.$ for $15 \mathrm{sec}, 60^{\circ} \mathrm{C}$ for $15 \mathrm{sec}$ and a gradual increment of 478 temperature with a ramp rate of $1 \%$ up to $95^{\circ} \mathrm{C}$ ) was programmed at the end of the RT-qPCR 479 to assess the specificity of the reactions. The genes ISYNA2 and GRP137 were selected as 480 endogenous controls following the stability values after a GeNorm pilot experiment. Their 481 stability was determined considering a GeNorm $\mathrm{M}$ value $<0.5$. Relative expression values 482 were calculated using the ThermoFisher Cloud software (Applied Biosystems) applying the $4832^{-\Delta \Delta \mathrm{Ct}}$ method. The same software was used to compare the biological groups. Significance 484 was set at a P-value $<0.05$.

\section{Acknowledgements}

\section{Author contributions}

489 MG, AS, and AlC conceived and designed the experiments. MR and JR-G carried the 490 phenotypic analysis. MG performed sperm purifications. MG and MR carried the RNA 491 extractions. AnC designed and carried the RT-qPCR and their analyses. AnC and BC 492 performed Sanger Sequencing validation. MG made the bioinformatics, statistic analysis and 493 analyzed the data. MG and AlC wrote the manuscript. All authors discussed the data and read 494 and approved the contents of the manuscript.

496 Disclosure statement

497 No potential conflict of interest was reported by the authors. 498

\section{Availability of data}


500 The datasets generated and/or analysed during the current study are available at NCBI's

$501 \quad$ BioProject PRJNA520978.

502

\section{Funding}

504 This work was supported by the Spanish Ministry of Economy and Competitiveness

505 (MINECO) under grant AGL2013-44978-R and grant AGL2017-86946-R and by the

506 CERCA Programme/Generalitat de Catalunya. AGL2017-86946-R was also funded by the

507 Spanish State Research Agency (AEI) and the European Regional Development Fund

508 (ERDF). We thank the Agency for Management of University and Research Grants

509 (AGAUR) of the Generalitat de Catalunya (Grant Number 2017 SGR 1060). We also

510 acknowledge the support of the Spanish Ministry of Economy and Competitivity for the

511 Center of Excellence Severo Ochoa 2016-2019 (Grant Number SEV-2015-0533) grant

512 awarded to the Centre for Research in Agricultural Genomics (CRAG). MG acknowledges a

513 Ph.D. studentship from MINECO (Grant Number BES-2014-070560).

\section{References}

517 1. Avendaño S, Neeteson-van Nieuwenhoven A-M, Knap P. The role of sustainable 518 commercial pig and poultry breeding for food security. Animal Frontiers 2013; 3:52-

519 57. doi: 10.2527/af.2013-0008.

520 2. Swindle MM, Makin A, Herron AJ, Clubb FJ, Frazier KS. Swine as Models in 521 Biomedical Research and Toxicology Testing. Vet Pathol 2012; 49:344-356. doi: $10.1177 / 0300985811402846$.

523 3. Hirsh A. Male subfertility. BMJ 2003; 327:669-672. doi: 10.1136/bmj.327.7416.669.

524 4. Frankenhuis MT, Wensing CJ. Induction of spermatogenesis in the naturally $525 \quad$ cryptorchid pig. Fertil Steril 1979; 31:428-433.

526 5. Bernabo N, Tettamanti E, Russo V, Martelli A, Turriani M, Mattoli M, Barboni B. 527 Extremely low frequency electromagnetic field exposure affects fertilization outcome 
528 in swine animal model. Theriogenology 2010; 73:1293-1305. doi: 10.1016/j.theriogenology.2009.12.010.

530 6. Park KE, Kaucher AV, Powell A, Waqas MS, Sandmaier SE, Oatley MJ, Park CH, 531 Tibary A, Donovan DM, Blomberg LA, Lillico SG, Whitelaw CB, et al. Generation 532 of germline ablated male pigs by CRISPR/Cas9 editing of the NANOS2 gene. Sci 533 Rep 2017; 7:40176. doi: 10.1038/srep40176.

534 7. Farrell PB, Presicce GA, Brockett CC, Foote RH. Quantification of bull sperm 535 characteristics measured by computer-assisted sperm analysis (CASA) and the relationship to fertility. Theriogenology 1998; 49:871-879. doi: 10.1016/S0093-

538 8. Love CC. Relationship between sperm motility, morphology and the fertility of stallions. Theriogenology 2011; 76:547-557.

doi: 10.1016/j.theriogenology.2011.03.007.

541 9. Holt C, Holt WV, Moore HD, Reed HC, Curnock RM. Objectively measured boar sperm motility parameters correlate with the outcomes of on-farm inseminations: results of two fertility trials. J Androl 1997; 18:312-323.

11. Hirai M, Boersma A, Hoeflich A, Wolf E, Foll J, Aumuller R, Braun J. Objectively measured sperm motility and sperm head morphometry in boars (Sus scrofa): Relation to fertility and seminal plasma growth factors. J Androl 2001; 22:104-110.

12. Hirano Y, Shibahara H, Obara H, Suzuki T, Takamizawa S, Yamaguchi C, Tsunoda computer-aided sperm analysis (CASA) and fertilization rates in vitro. J Assist Reprod Gen 2001; 18:213-218.

556 14. Paston MJ, Sarkar S, Oates RP, Badawy SZA. Computer-Aided Semen Analysis Variables as Predictors of Male-Fertility Potential. Arch Andrology 1994; 33:93-99. doi: Doi 10.3109/01485019408987809.

13. Aitken RJ. Sperm function tests and fertility. Int J Androl 2006; 29:69-74. doi: 10.1111/j.1365-2605.2005.00630.x.

15. Jodar M, Kalko S, Castillo J, Ballesca JL, Oliva R. Differential RNAs in the sperm cells of asthenozoospermic patients. Hum Reprod 2012; 27:1431-1438. doi: 10.1093/humrep/des021. 
562 16. Pelloni M, Paoli D, Majoli M, Pallotti F, Carlini T, Lenzi A, Lombardo F. Molecular study of human sperm RNA: Ropporin and CABYR in asthenozoospermia. J Endocrinol Invest 2018; 41:781-787. doi: 10.1007/s40618-017-0804-x.

17. Parthipan S, Selvaraju S, Somashekar L, Arangasamy A, Sivaram M, Ravindra JP. Spermatozoal transcripts expression levels are predictive of semen quality and conception rate in bulls (Bos taurus). Theriogenology 2017; 98:41-49. doi: 10.1016/j.theriogenology.2017.04.042.

18. Salas-Huetos A, Blanco J, Vidal F, Godo A, Grossmann M, Pons MC, S FF, Garrido $\mathrm{N}$, Anton E. Spermatozoa from patients with seminal alterations exhibit a differential

19. Capra E, Turri F, Lazzari B, Cremonesi P, Gliozzi TM, Fojadelli I, Stella A, Pizzi F.

20. Jeck WR, Sharpless NE. Detecting and characterizing circular RNAs. Nat Biotechnol

21. Abe N, Abe H, Ito Y. Dumbbell-shaped nanocircular RNAs for RNA interference. J

22. Cocquerelle C, Mascrez B, Hetuin D, Bailleul B. Mis-splicing yields circular RNA molecules. FASEB J 1993; 7:155-160.

23. Cortes-Lopez M, Miura P. Emerging Functions of Circular RNAs. Yale J Biol Med 2016; 89:527-537.

25. Guo JU, Agarwal V, Guo HL, Bartel DP. Expanded identification and

24. Westholm JO, Miura P, Olson S, Shenker S, Joseph B, Sanfilippo P, Celniker SE, Graveley BR, Lai EC. Genome-wide Analysis of Drosophila Circular RNAs Reveals Their Structural and Sequence Properties and Age-Dependent Neural Accumulation. Cell Rep 2014; 9:1966-1980. doi: 10.1016/j.celrep.2014.10.062.

characterization of mammalian circular RNAs. Genome Biol 2014; 15. doi: 10.1186/s13059-014-0409-z.

26. Memczak S, Jens M, Elefsinioti A, Torti F, Krueger J, Rybak A, Maier L, Mackowiak SD, Gregersen LH, Munschauer M, Loewer A, Ziebold U, et al. Circular RNAs are a large class of animal RNAs with regulatory potency. Nature 2013; 495:333-338. doi: 10.1038/nature11928. 
596 27. Xia SY, Feng J, Lei LJ, Hu J, Xia LJ, Wang J, Xiang Y, Liu LJ, Zhong S, Han L, He CJ. Comprehensive characterization of tissue-specific circular RNAs in the human and mouse genomes. Brief Bioinform 2017; 18:984-992. doi: 10.1093/bib/bbw081.

28. Liang GM, Yang YL, Niu GL, Tang ZL, Li K. Genome-wide profiling of Sus scrofa circular RNAs across nine organs and three developmental stages. DNA Res 2017; 24:523-535. doi: 10.1093/dnares/dsx022.

30. Zhang Z, Yang T, Xiao J. Circular RNAs: Promising Biomarkers for Human Diseases.

31. Quan GB, Li JL. Circular RNAs: biogenesis, expression and their potential roles in reproduction. J Ovarian Res 2018; 11. doi: 10.1186/s13048-018-0381-4.

32. Cheng J, Huang J, Yuan S, Zhou S, Yan W, Shen W, Chen Y, Xia X, Luo A, Zhu D,

33. Qian YT, Lu YQ, Rui C, Qian YJ, Cai MH, Jia RZ. Potential Significance of Circular Wang S. Circular RNA expression profiling of human granulosa cells during maternal aging reveals novel transcripts associated with assisted reproductive technology outcomes. Plos One 2017; 12:e0177888. doi: 10.1371/journal.pone.0177888.

36. Glazar P, Papavasileiou P, Rajewsky N. circBase: a database for circular RNAs. RNA

37. Rybak-Wolf A, Stottmeister C, Glazar P, Jens M, Pino N, Giusti S, Hanan M, Behm RNA in Human Placental Tissue for Patients with Preeclampsia. Cell Physiol

34. Gòdia M, Estill M, Castelló A, Balasch S, Rodríguez-Gil JE, Krawetz SA, Sánchez A, Clop A. A RNA-seq analysis to describe the boar sperm transcriptome and its seasonal changes. Front Genet 2018. doi: 10.3389/fgene.2019.00299.

35. Gòdia M, Swanson G, Krawetz SA. A history of why fathers' RNA matters. Biol Reprod 2018; 99:147-159. doi: 10.1093/biolre/ioy007. Mammalian Brain Are Highly Abundant, Conserved, and Dynamically Expressed. Mol Cell 2015; 58:870-885. doi: 10.1016/j.molcel.2015.03.027.

38. Jeck WR, Sorrentino JA, Wang K, Slevin MK, Burd CE, Liu J, Marzluff WF, Sharpless NE. Circular RNAs are abundant, conserved, and associated with ALU repeats. RNA 2013; 19:141-157. doi: 10.1261/rna.035667.112. 
630 39. Salzman J, Chen RE, Olsen MN, Wang PL, Brown PO. Cell-type specific features of circular RNA expression. PLoS Genet 2013; 9:e1003777. doi: 10.1371/journal.pgen.1003777.

633 40. Zhang Y, Zhang XO, Chen T, Xiang JF, Yin QF, Xing YH, Zhu S, Yang L, Chen LL. Circular intronic long noncoding RNAs. Mol Cell 2013; 51:792-806. doi: 10.1016/j.molcel.2013.08.017.

42. Gruner H, Cortes-Lopez M, Cooper DA, Bauer M, Miura P. CircRNA accumulation

43. Banaszewska D, Kondracki S. An Assessment of the Breeding Maturity of doi: 10.3409/fb60_3-4.151-162.

45. Marjanovic M, Sanchez-Huertas C, Terre B, Gomez R, Scheel JF, Pacheco S, Knobel

46. Ota K, Jaiswal MK, Ramu S, Jeyendran R, Kwak-Kim J, Gilman-Sachs A, Beaman

47. Asghari A, Marashi SA, Ansari-Pour N. A sperm-specific proteome-scale metabolic KD. Expression of a2 Vacuolar ATPase in Spermatozoa is Associated with Semen 
662 49. Aitken RJ, Baker MA, Nixon B. Are sperm capacitation and apoptosis the opposite ends of a continuum driven by oxidative stress? Asian J Androl 2015; 17:633-639. doi: 10.4103/1008-682X.153850.

50. Yanagiya A, Delbes G, Svitkin YV, Robaire B, Sonenberg N. The poly(A)-binding protein partner Paip2a controls translation during late spermiogenesis in mice. J Clin Invest 2010; 120:3389-3400. doi: 10.1172/JCI43350.

51. Schwab KR, Smith GD, Dressler GR. Arrested spermatogenesis and evidence for DNA damage in PTIP mutant testes. Dev Biol 2013; 373:64-71. doi: 10.1016/j.ydbio.2012.10.006.

53. Luo JP, McGinnis LK, Carlton C, Beggs HE, Kinsey WH. PTK2b function during

54. Mi YJ, Shi Z, Li J. Spata19 Is Critical for Sperm Mitochondrial Function and Male

55. Rigau T, Rivera M, Palomo MJ, Fernandez-Novell JM, Mogas T, Ballester J, Pena A, Otaegui PJ, Guinovart JJ, Rodríguez-Gil JE. Differential effects of glucose and fructose on hexose metabolism in dog spermatozoa. Reproduction 2002; 123:579-591. doi: DOI 10.1530/rep.0.1230579.

56. Sendler E, Johnson GD, Mao SH, Goodrich RJ, Diamond MP, Hauser R, Krawetz SA. Stability, delivery and functions of human sperm RNAs at fertilization. Nucleic Acids Res 2013; 41:4104-4117. doi: 10.1093/nar/gkt132.

58. Oatley JM, Brinster RL. Regulation of spermatogonial stem cell self-renewal in

mammals. Annu Rev Cell Dev Biol 2008; 24:263-286. doi: 10.1146/annurev.cellbio.24.110707.175355. 
695 59. Franca LR, Silva VA, Jr., Chiarini-Garcia H, Garcia SK, Debeljuk L. Cell

696

697

698

699

700

701

702

703

704

705

706

707

708

709

710

711

712

713

714

715

716

717

718

719

720

721

722

723

724

725

726

proliferation and hormonal changes during postnatal development of the testis in the pig. Biol Reprod 2000; 63:1629-1636.

60. Jumeau F, Chalmel F, Fernandez-Gomez FJ, Carpentier C, Obriot H, Tardivel M, Caillet-Boudin ML, Rigot JM, Rives N, Buee L, Sergeant N, Mitchell V. Defining the human sperm microtubulome: an integrated genomics approach. Biol Reprod 2017; 96:93-106. doi: 10.1095/biolreprod.116.143479.

61. Abu-Halima M, Hammadeh M, Schmitt J, Leidinger P, Keller A, Meese E, Backes C. Altered microRNA expression profiles of human spermatozoa in patients with different spermatogenic impairments. Fertil Steril 2013; 99:1249-1255.e1216. doi: 10.1016/j.fertnstert.2012.11.054.

62. Muñoz X, Mata A, Bassas L, Larriba S. Altered miRNA Signature of Developing Germ-cells in Infertile Patients Relates to the Severity of Spermatogenic Failure and Persists in Spermatozoa. Sci Rep 2015; 5. doi: 10.1038/srep17991.

63. Bolusani S, Young BA, Cole NA, Tibbetts AS, Momb J, Bryant JD, Solmonson A, Appling DR. Mammalian MTHFD2L encodes a mitochondrial methylenetetrahydrofolate dehydrogenase isozyme expressed in adult tissues. J Biol Chem 2011; 286:5166-5174. doi: 10.1074/jbc.M110.196840.

64. Wallock L, Jacob R, Woodall A, Ames B. Nutritional status and positive relation of plasma folate to fertility indices in nonsmoking men. FASEB J 1997; 11:1068-1068.

65. Li FQ, Chen X, Fisher C, Siller SS, Zelikman K, Kuriyama R, Takemaru KI. BAR Domain-Containing FAM92 Proteins Interact with Chibby1 To Facilitate Ciliogenesis. Mol Cell Biol 2016; 36:2668-2680. doi: 10.1128/MCB.00160-16.

66. Suchocki T, Szyda J. Genome-wide association study for semen production traits in Holstein-Friesian bulls. J Dairy Sci 2015; 98:5774-5780. doi: 10.3168/jds.2014-8951.

67. Ma JD, Fan Y, Zhang JW, Feng SY, Hu ZH, Qiu WL, Long KR, Jin L, Tang QZ, Wang X, Zhou Q, Gu YR, et al. Testosterone-Dependent miR-26a-5p and let-7g-5p Act as Signaling Mediators to Regulate Sperm Apoptosis via Targeting PTEN and PMAIP1. Int J Mol Sci 2018; 19. doi: 10.3390/ijms 19041233.

68. Bao X, Tang J, Lopez-Pajares V, Tao S, Qu K, Crabtree GR, Khavari PA. ACTL6a enforces the epidermal progenitor state by suppressing SWI/SNF-dependent induction of KLF4. Cell Stem Cell 2013; 12:193-203. doi: 10.1016/j.stem.2012.12.014. 
69. Alsheimer M, Drewes T, Schutz W, Benavente R. The cancer/testis antigen CAGE-1 is a component of the acrosome of spermatids and spermatozoa. Eur J Cell Biol 2005; 84:445-452. doi: 10.1016/j.ejcb.2004.11.003.

70. Zhou R, Wang R, Qin Y, Ji J, Xu M, Wu W, Chen M, Wu D, Song L, Shen H, Sha J, Miao D, et al. Mitochondria-related miR-151a-5p reduces cellular ATP production by targeting CYTB in asthenozoospermia. Sci Rep 2015; 5:17743. doi: 10.1038/srep17743.

71. Marin-Briggiler CI, Jha KN, Chertihin O, Buffone MG, Herr JC, Vazquez-Levin MH, Visconti PE. Evidence of the presence of calcium/calmodulin-dependent protein kinase IV in human sperm and its involvement in motility regulation. J Cell Sci 2005; 118:2013-2022. doi: 10.1242/jcs.02326.

72. Liu Y, DeBoer K, de Kretser DM, O'Donnell L, O'Connor AE, Merriner DJ, Okuda H, Whittle B, Jans DA, Efthymiadis A, McLachlan RI, Ormandy CJ, et al. LRGUK-1 Is Required for Basal Body and Manchette Function during Spermatogenesis and Male Fertility. PLoS Genet 2015; 11. doi: 10.1371/journal.pgen.1005090.

73. Ehrmann I, Dalgliesh C, Liu Y, Danilenko M, Crosier M, Overman L, Arthur HM, Lindsay S, Clowry GJ, Venables JP, Fort P, Elliott DJ. The tissue-specific RNA binding protein T-STAR controls regional splicing patterns of neurexin pre-mRNAs in the brain. PLoS Genet 2013; 9:e1003474. doi: 10.1371/journal.pgen.1003474.

74. Ferlin A, Raicu F, Gatta V, Zuccarello D, Palka G, Foresta C. Male infertility: role of genetic background. Reprod Biomed Online 2007; 14:734-745.

75. Gadea J. Sperm factors related to in vitro and in vivo porcine fertility. Theriogenology 2005; 63:431-444. doi: 10.1016/j.theriogenology.2004.09.023.

76. $\mathrm{R}$ Developmental Core Team. R: A language and environment for statistical computing. 2010.

77. Gòdia M, Mayer FQ, Nafissi J, Castelló A, Rodríguez-Gil JE, Sánchez A, Clop A. A technical assessment of the porcine ejaculated spermatozoa for a sperm-specific RNA-seq analysis. Syst Biol Reprod Med 2018; 64:291-303. doi: 10.1080/19396368.2018.1464610.

78. Bolger AM LM, Usadel B. Trimmomatic: a flexible trimmer for Illumina sequence data. Bioinformatics 2014; 30:2114-2120. doi: 10.1093/bioinformatics/btu170.

79. Quinlan AR, Hall IM. BEDTools: a flexible suite of utilities for comparing genomic features. Bioinformatics 2010; 26:841-842. doi: 10.1093/bioinformatics/btq033. 
760 80. Kuhn RM HD, Kent WJ. . The UCSC genome browser and associated tools Brief Bioinform 2013; 14:144-161. doi: doi: 10.1093/bib/bbs038.

81. Martin M. Cutadapt removes adapter sequences from high-throughput sequencing reads. EMBnet J 2011; 17:10-12. doi: 10.14806/ej.17.1.200.

82. Rueda A, Barturen G, Lebron R, Gomez-Martin C, Alganza A, Oliver JL, Hackenberg M. sRNAtoolbox: an integrated collection of small RNA research tools. Nucleic Acids Res 2015; 43:W467-473. doi: 10.1093/nar/gkv555.

83. Kozomara A G-JS. miRBase: integrating microRNA annotation and deep-sequencing data. Nucleic Acids Res 2011; 39:D152-D157. doi: 10.1093/nar/gkq1027.

84. Reverter A, Chan EK. Combining partial correlation and an information theory

87. Mi H, Dong Q, Muruganujan A, Gaudet P, Lewis S, Thomas PD. PANTHER version approach to the reversed engineering of gene co-expression networks. Bioinformatics 2008; 24:2491-2497. doi: 10.1093/bioinformatics/btn482.

85. Enright AJ, John B, Gaul U, Tuschl T, Sander C, Marks DS. MicroRNA targets in Drosophila. Genome Biol 2003; 5:R1. doi: 10.1186/gb-2003-5-1-r1.

86. Shannon P, Markiel A, Ozier O, Baliga NS, Wang JT, Ramage D, Amin N, Schwikowski B, Ideker T. Cytoscape: A software environment for integrated models of biomolecular interaction networks. Genome Res 2003; 13:2498-2504. doi: 
Table 1. Top 20 most abundant circRNAs in swine sperm. circRNA genomic coordinates are indicated in the format chromosome:start_position..end_position. Mean and SD: standard deviation, are in CPM (Counts Per Million).

\begin{tabular}{|c|c|c|c|c|c|c|}
\hline $\begin{array}{l}\text { circRNA } \\
\text { name }\end{array}$ & circRNA genomic coordinates & Mean & SD & circRNA type & Ensembl ID & $\begin{array}{l}\text { Host gene } \\
\text { symbol }\end{array}$ \\
\hline$\overline{\text { ssc_circ_0493 }}$ & $13: 75694364 . .75694548$ & 136.4 & 80.7 & intronic & ENSSSCG00000011645 & CEP63 \\
\hline ssc_circ_0220 & 11:17636879..17653264 & 128.2 & 52.5 & intergenic & & \\
\hline ssc_circ_0475 & 13:61967873..61969753 & 100.2 & 56.8 & lincRNA & ENSSSCG00000035295 & \\
\hline ssc_circ_1097 & $4: 590305 . .590427$ & 75.8 & 51.9 & $\mathrm{CDS}$ & ENSSSCG00000005916 & WDR97 \\
\hline ssc_circ_1141 & $5: 22008783 . .22009750$ & 75.0 & 35.4 & CDS & ENSSSCG00000000406 & PTGES3 \\
\hline ssc_circ_1062 & 4:130322062..130339222 & 62.2 & 33.1 & $\mathrm{CDS}$ & ENSSSCG00000006939 & ZNHIT6 \\
\hline ssc_circ_0537 & $14: 29281863 . .29290551$ & 59.9 & 34.8 & $\mathrm{CDS}$ & ENSSSCG00000009766 & ATP6VOA2 \\
\hline ssc_circ_0777 & $18: 14243637 . .14259662$ & 47.3 & 23.9 & CDS & ENSSSCG00000016529 & $A G B L 3$ \\
\hline ssc_circ_0860 & 2:3096936...3098647 & 45.1 & 35.6 & CDS & ENSSSCG00000037451 & PPFIAI \\
\hline ssc_circ_0805 & 18:54260018..54270584 & 40.0 & 52.3 & $\mathrm{CDS}$ & ENSSSCG00000035581 & $S U G C T$ \\
\hline ssc_circ_0132 & $1: 641933 . .653683$ & 33.2 & 19.1 & CDS & ENSSSCG00000004008 & WDR27 \\
\hline ssc_circ_1452 & $8: 56584279 . .56590933$ & 28.7 & 21.9 & intergenic & & \\
\hline ssc_circ_1575 & AEMK02000682.1:1719140..1720285 & 28.4 & 24.6 & $\mathrm{CDS}$ & ENSSSCG00000005753 & CAMSAPl \\
\hline ssc_circ_1413 & 8:116275909..116294261 & 26.9 & 15.5 & CDS & ENSSSCG00000022788 & $P P A 2$ \\
\hline ssc_circ_0895 & $2: 64840342 . .64840811$ & 25.8 & 17.5 & 5' UTR & ENSSSCG00000013776 & $D D X 39 A$ \\
\hline ssc_circ_0102 & 1:264102211..264103554 & 24.3 & 22.1 & intronic & ENSSSCG00000005582 & STRBP \\
\hline
\end{tabular}


ssc_circ_0363 13:119893907..119895097

ssc_circ_1101 4:6367950..6392035

ssc_circ_0839 2:141254413..141254577

ssc_circ_0795 18:3101570..3102902
$24.2 \quad 14.9 \quad$ inetergenic

$\begin{array}{lllll}22.6 & 28.8 & \text { CDS } & \text { ENSSSCG00000005941 KHDRBS3 }\end{array}$

21.820 .9 CDS + 5' UTR ENSSSCG00000026606 PAIP2

$\begin{array}{lllll}21.7 & 9.0 & \text { CDS } & \text { ENSSSCG00000025221 PAXIP1 }\end{array}$ 
Table 2. circRNA hotspot genes in swine sperm. 12 genes presented 5 or more different exonic circRNAs.

\begin{tabular}{lll}
\hline Gene symbol & Ensembl ID & Number of circRNAs \\
\hline DENND1B & ENSSSCG00000010900 & 8 \\
DENND1A & ENSSSCG00000005585 & 7 \\
TESK2 & ENSSSCG00000003917 & 6 \\
UIMC1 & ENSSSCG00000022508 & 6 \\
ARMC9 & ENSSSCG00000023994 & 5 \\
CAMSAP1 & ENSSSCG00000005753 & 5 \\
KDM5B & ENSSSCG00000010928 & 5 \\
PTK2 & ENSSSCG00000038397 & 5 \\
RPS6KC1 & ENSSSCG00000015586 & 5 \\
SPATA19 & ENSSSCG00000025612 & 5 \\
SLC5A10 & ENSSSCG00000018049 & 5 \\
$W N K 1$ & ENSSSCG00000000753 & 5 \\
\hline
\end{tabular}

807

808

809

810

811

812

813

814

815

816 
Table 3. Concordance between the boar sperm circRNAs and other tissues. Number of circRNAs according to Sscrofa11.1 liftover, in human, mice and swine (detailed by tissue). The column $\%$ swine sperm shows the proportion of swine sperm circRNAs that were identified in the given species and tissues. Column $\%$ of the total circRNAs lists the proportion of circRNAs from that tissue or species that found a homolog in the boar sperm.

\begin{tabular}{lcccc}
\hline Species / Tissues & Number of circRNAs & \% swine sperm & \% of the total circRNAs \\
\hline Human & 90,067 & $24.0 \%$ & $0.4 \%$ & \\
Mice & 15,498 & $11.3 \%$ & $1.2 \%$ & \\
Swine & 6,663 & $20.3 \%$ & $4.9 \%$ & \\
\multicolumn{1}{c}{ Basal ganglia } & 456 & $2.6 \%$ & & $9.2 \%$ \\
Brain stem & 820 & $5.3 \%$ & $10.2 \%$ \\
Cerebellum & 1,061 & $5.6 \%$ & $8.4 \%$ \\
Cortex & 2,163 & $11.3 \%$ & $8.4 \%$ \\
Hippocampus & 549 & $3.2 \%$ & $9.3 \%$ \\
Fat & 494 & $2.4 \%$ & $7.7 \%$ \\
Heart & 539 & $2.2 \%$ & $6.5 \%$ \\
Kidney & 469 & $1.9 \%$ & $6.4 \%$ \\
Liver & 353 & $2.1 \%$ & $9.3 \%$ \\
Lung & 683 & $2.7 \%$ & $6.3 \%$ \\
Muscle & 532 & $2.6 \%$ & $7.9 \%$ \\
Ovarium & 652 & $3.8 \%$ & $9.4 \%$ \\
Spleen & 241 & $1.4 \%$ & $9.1 \%$ \\
Testes & 2,685 & $11.6 \%$ & \\
\hline
\end{tabular}




\section{Figure legends}

839 Figure 1. Characteristics of the genomic features of the sperm circRNAs identified in 840 the boar sperm.

841 A. Distribution of genomic location (CDS, intergenic, intronic, 3' UTR or 5'UTR) of the

8421,598 circRNAs identified in boar sperm. B. Distribution of the number of exons forming the

843 exonic circRNAs. C. Distribution of the nucleotide length of the exonic circRNAs

845 Figure 2. circRNA-miRNA interaction network. circRNA:miRNA relationships predicted

846 by both experimental data and miRNA target prediction in circRNAs. Circular and square

847 nodes represent circRNAs and miRNAs, respectively. The node and letter sizes indicate the 848 number of significant correlations involving the node.

850 Figure 3. Validation of circRNAs that displayed RNA-seq abundance correlation with sperm motility.

852 A. Sanger sequencing validation of the circRNAs black splice junction for ssc_circ_1132

853 from LIN7A, ssc_circ_1458 from LRBA and ssc_circ_1321 from PAPOLA. B. Relative

854 abundance of six circRNAs in samples with extreme and divergent motility values (18 high

855 and 18 low) obtained by RT-qPCR. The RNA-seq association between ssc_circ_1458 and 856 ssc_circ_1321 and sperm motility was validated by RT-qPCR. 
858 Supplementary Figure 1. Validation of the amplified set of circRNAs by agarose-gel. A. 859 Amplification of the 2 randomly selected circRNAs. Two different primer sets for 860 ssc_circ_1141 from PTGES3 were tested (primer pair a in lane 2 and b in lanes 3 and 4) and 861 ssc_circ_0670 from BAZ2B (lane 5). B. Validation of the circRNAs correlated to sperm 862 motility parameters: ssc_circ_1321 from PAPOLA (ENSSSCG00000002505), ssc_circ_1458

863 from $L R B A$, ssc_circ_0437 from $U L K 4$, two different primer sets for ssc_circ_1061 from 864 ZNHIT6 primer pair a and b, ssc_circ_1132 from LIN7A, two different primer sets for 865 ssc_circ_1101 from KHDRBS3, primer pair a (which resulted in amplification of two splicing 866 forms and was excluded) and b, and ssc_circ_0118 from PDE10A that resulted in 867 amplification of two splicing forms (and excluded from further analysis).

869 Supplementary Figure 2. Sanger sequencing validation of the set of circRNAs. A. 870 ssc_circ_1141 from PTGES3. B. ssc_circ_0670 from BAZ2B. C. ssc_circ_0437 from ULK4. D. ssc_circ_1061 from ZNHIT6. E. ssc_circ_1101 from KHDRBS3. 
888 Supplementary Tables

889 Supplementary Table 1. List of the 1,598 circRNAs identified in sperm with coordinates of

890 the exonic host gene and the mean and Standard Deviation (SD) of the RNA abundances (in

891 CPM) in the 40 samples.

892

893 Supplementary Table 2. Gene Ontology analysis and FDR value of the circRNA host genes.

895 Supplementary Table 3. Correlation of circRNAs with sperm motility parameters. Including

896 the exonic host gene, if tested for validation and article reference when the host gene has

897 been associated to sperm biology or male fertility. MT: total percentage of motile cells; VCL:

898 curvilinear velocity; VSL: straight line velocity; VAP: velocity of the sperm cells; ns: not 899 significant.

900

901 Supplementary Table 4. Comparison across swine circRNAs tissues.

902

903 Supplementary Table 5. CircRNA and reference genes primers designed. 
$63.6 \% \mathrm{CDS}$

$13.5 \%$ Intergenic

$4.4 \%$ Intronic

$0.4 \%$ 3' UTR

$1.2 \% 5^{\prime}$ UTR + CDS + 3'UTR

$3.0 \% \mathrm{CDS}+3^{\prime} \mathrm{UTR}$

$14.0 \% \mathrm{CDS}+5^{\prime} \mathrm{UTR}$

$$
\text { (1) }
$$

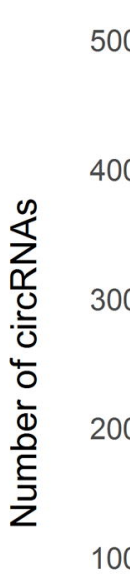

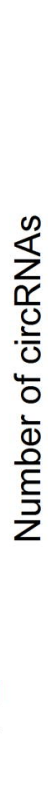

Number of exons

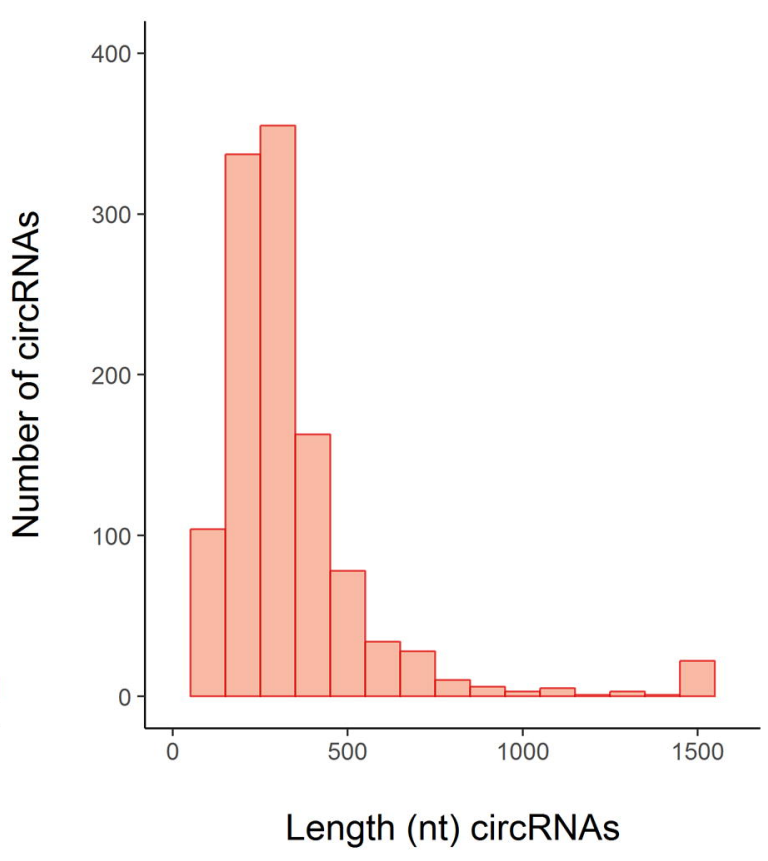




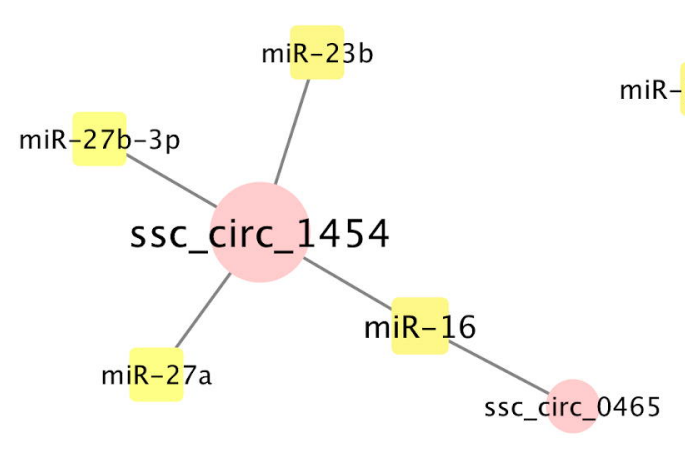

miR-146a-5p

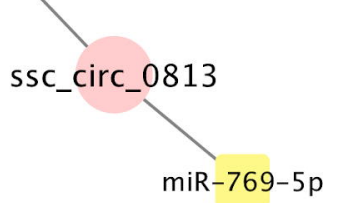

miR-769-5p

SSC_circ_0571 ssc circ 0041

ssc_circ_0314

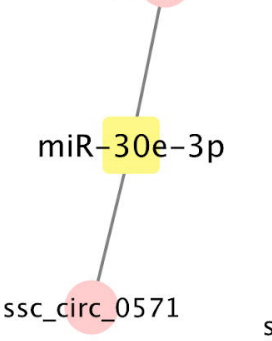

Ssc_circ_0767

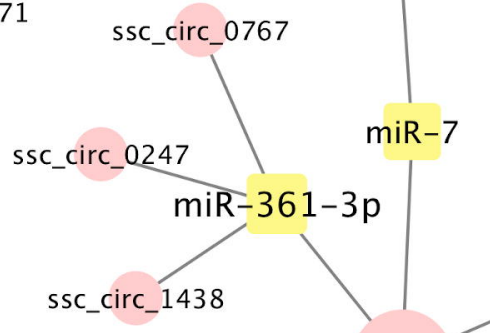

Ssc_circ_0174

miR-186

SsC_circ_0984

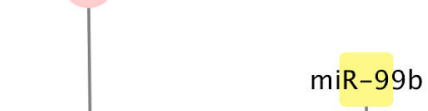

miR-148b-3p

miR-128
SsC_circ_0707

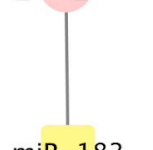

SSC_circ_0442

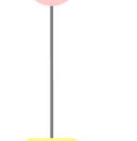

miR-7134-3p
SsC_circ_0198

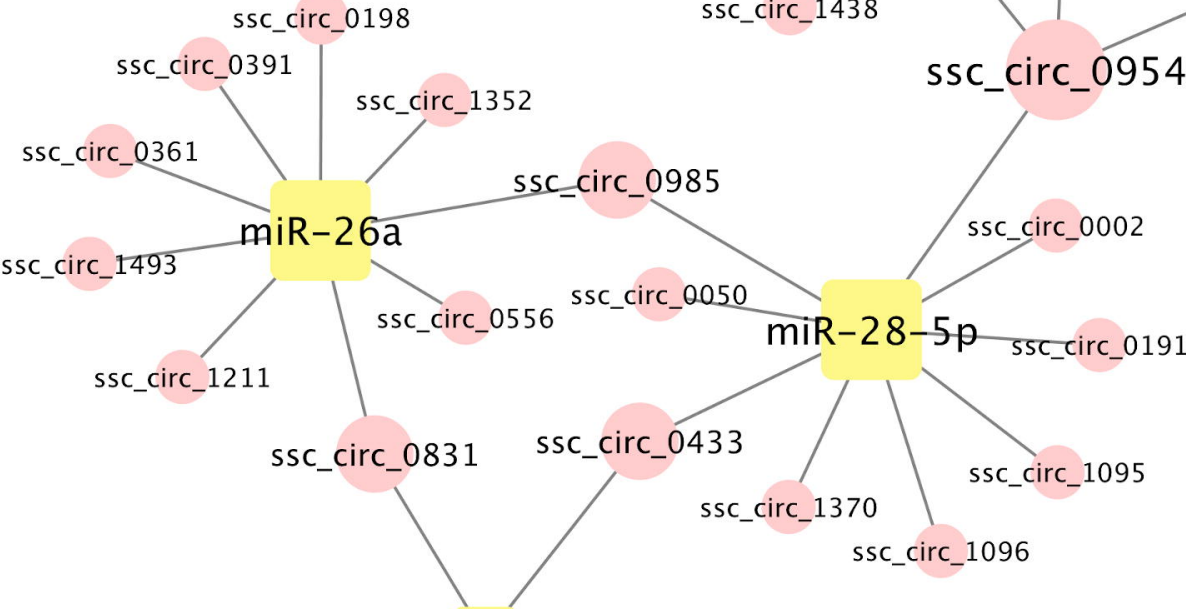

miR $-140-3 p$

ssc_circ_1117

miR-664-3p

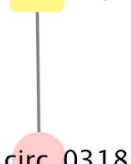

SsC_circ_0318

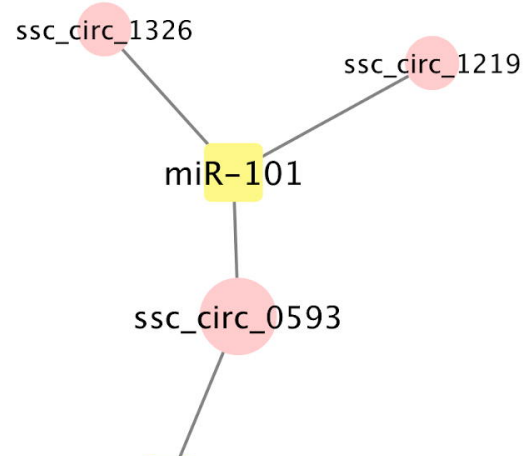

miR-194b-5p

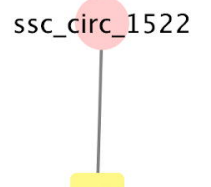

$m i R-499-5 p \quad m i R-486 \quad m i R-342 \quad m i R-28-3 p$
SsC_circ 0759

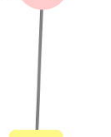

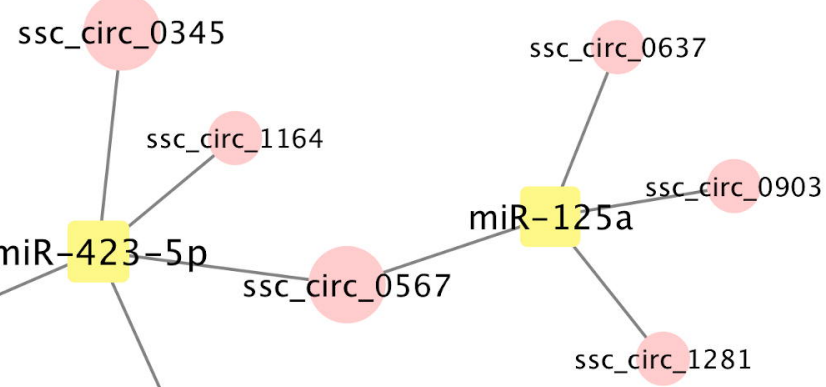

SsC_circ_1437

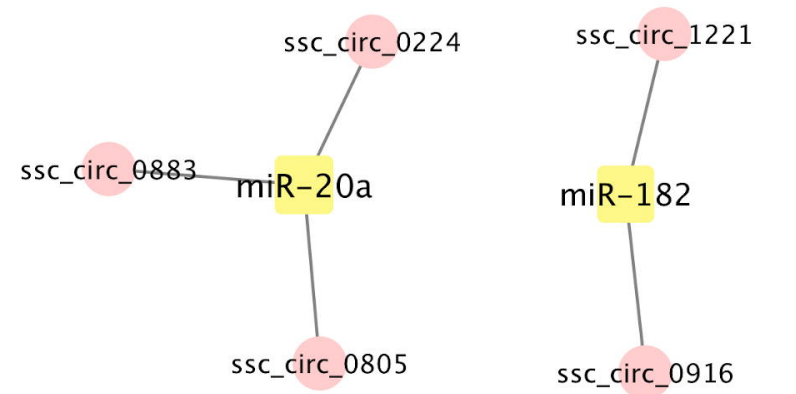


A

bioRxiv preprint doj: https://doi.org/10.1101/608026; this version posted April 16, 2019. The copyright holder for this preprint (which was not

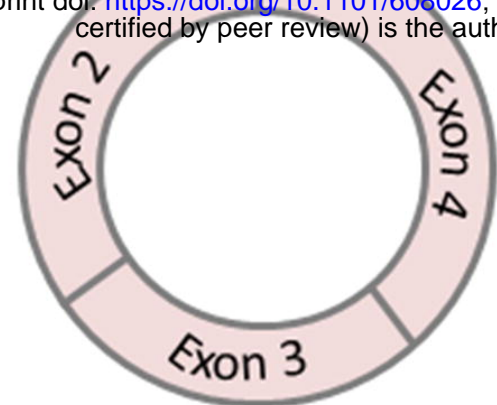

Back-spliced junction

$\nabla$

T G AACGGAG TG A TG TTGCCAGAG

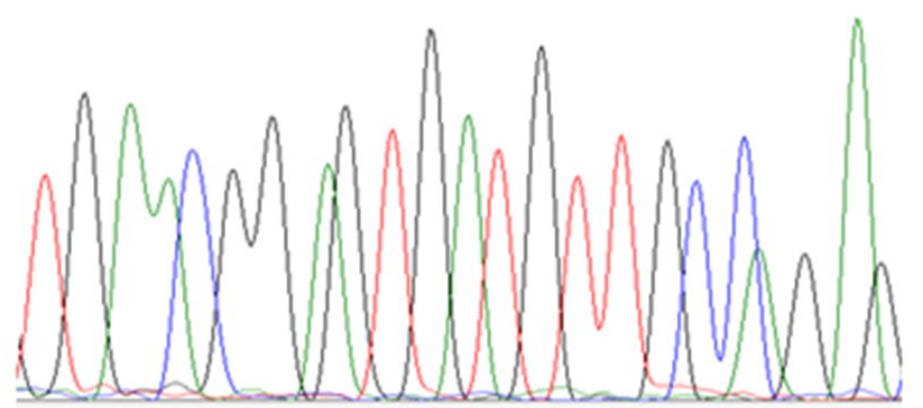

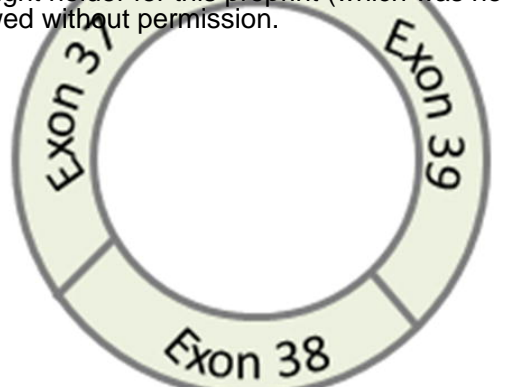

Back-spliced junction
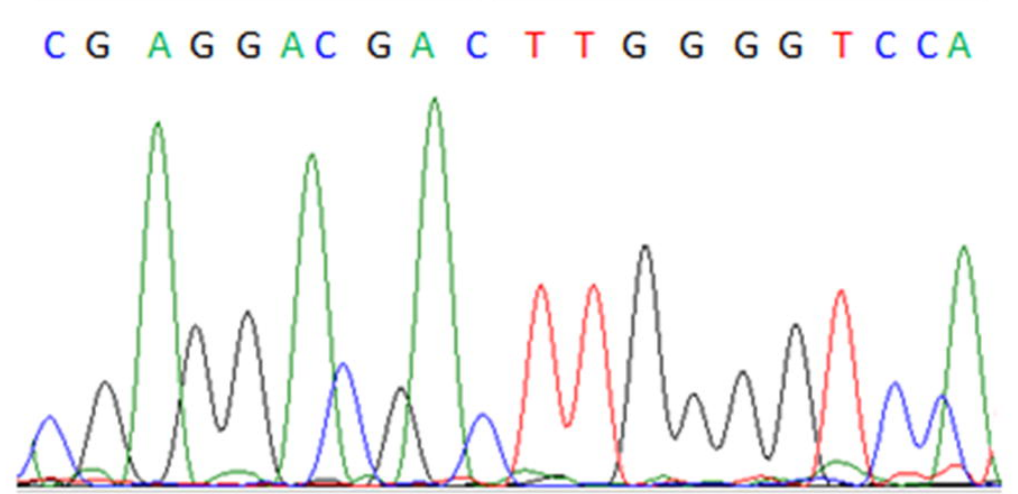

Back-spliced junction
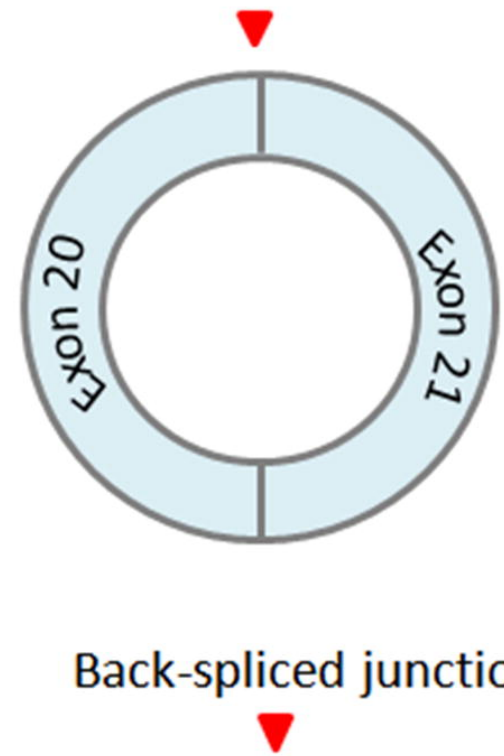

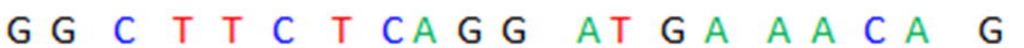

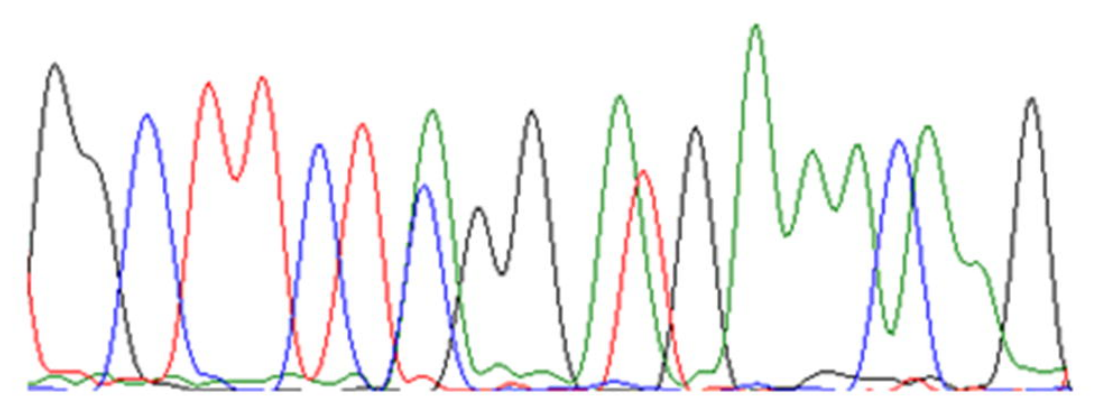

B

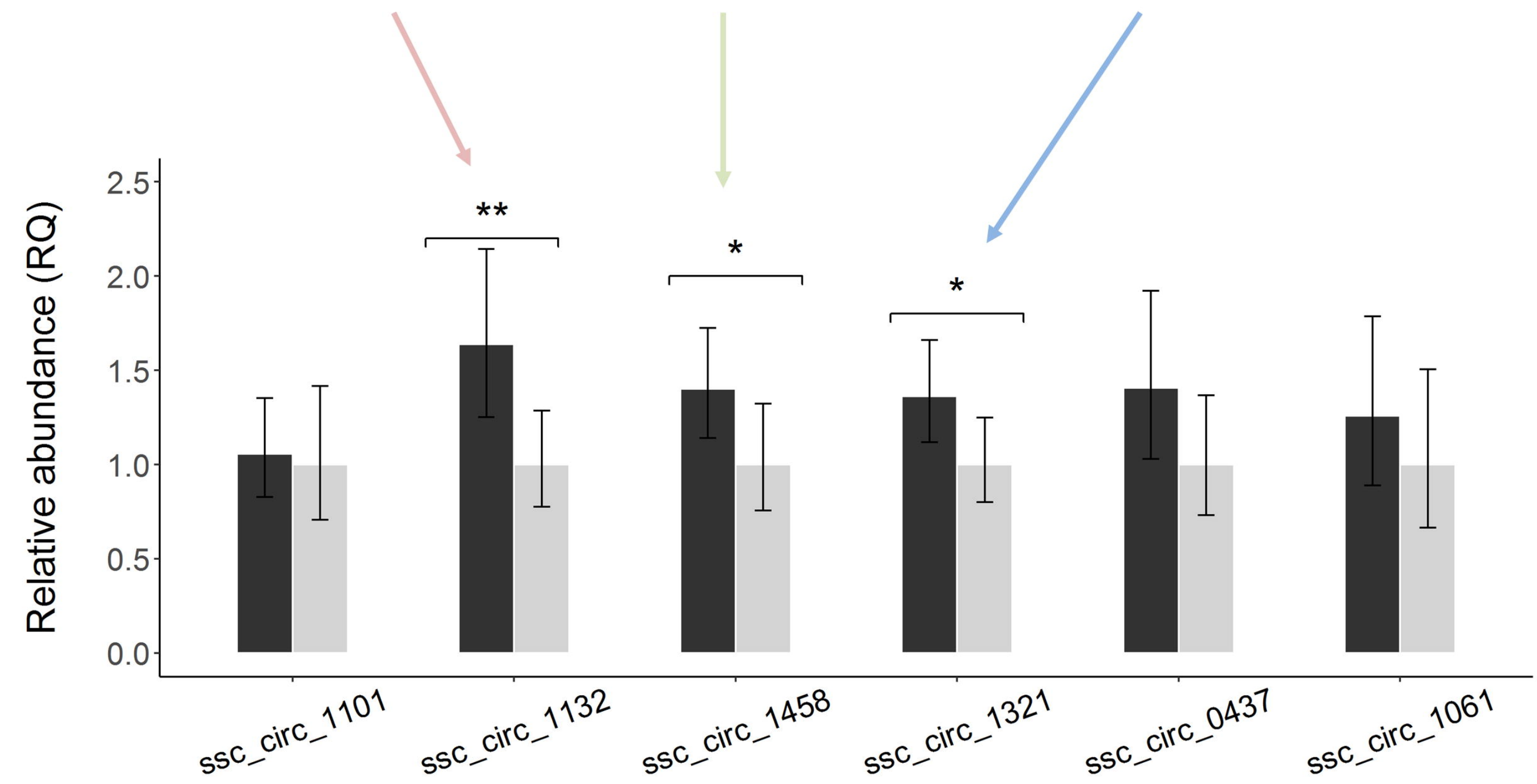

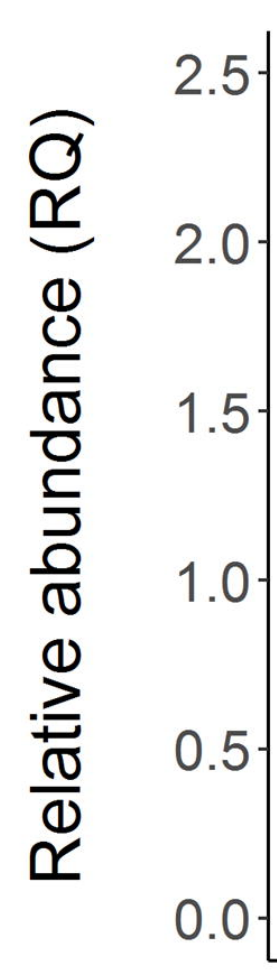

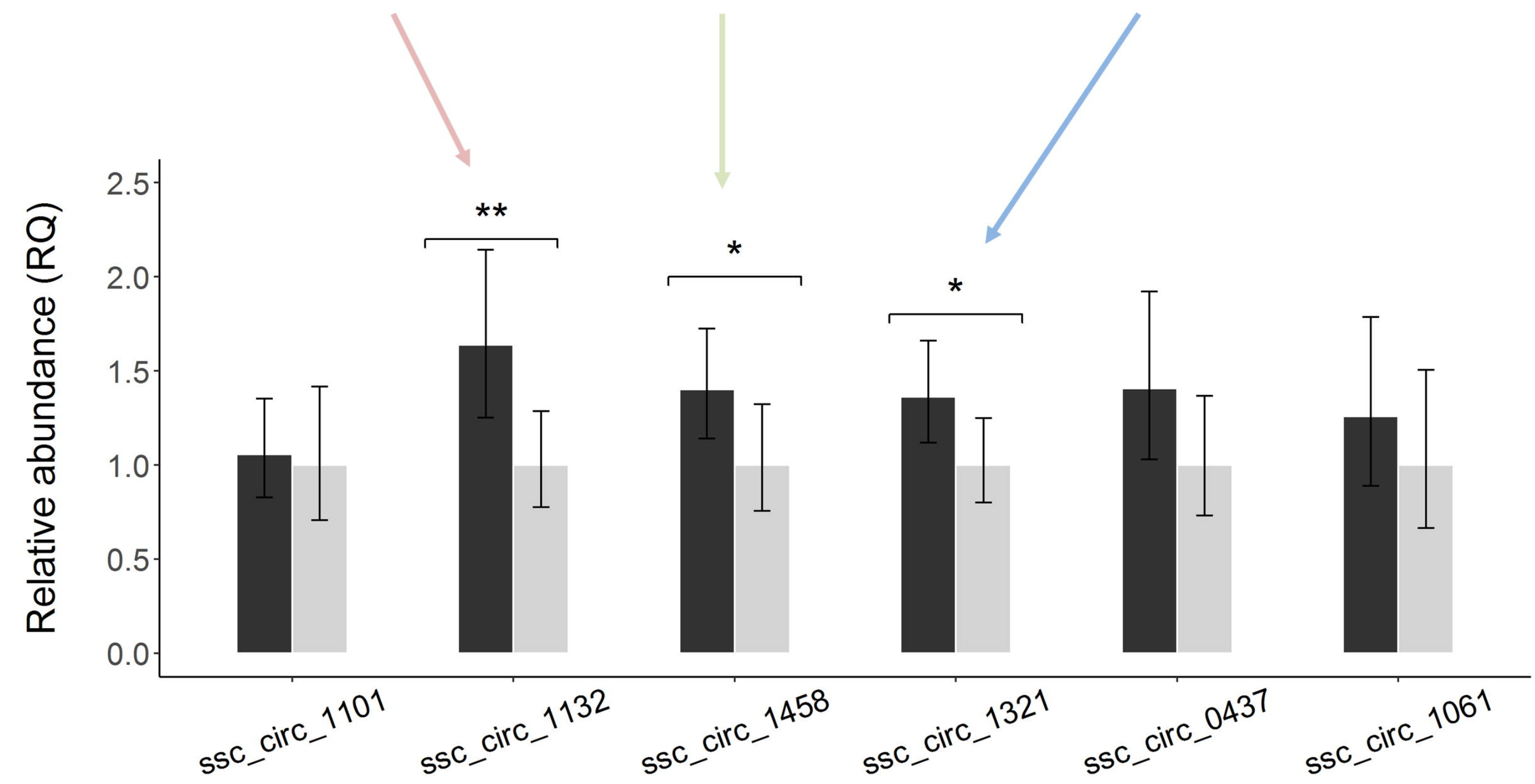

High

Low 